\title{
EPJ Web of Conferences : Application du concept « Lean Manufacturing » dans un atelier de fabrication moteur.
}

\author{
Florian BONZON \\ PSA Peugeot Citroën Automobiles, DRD/DCTC/CIMB/CIUO/ITPU/UPQM, 18 rue des Fauvelles 92256 La Garenne- \\ Colombes Cedex, FRANCE
}

\begin{abstract}
In 2011, PSA launched a brand new 3 cylinder petrol engine in the Tremery plant. A new workshop was completely designed according to the Lean Manufacturing concepts. Indeed the metrology room was thus designed according the principles of just in time, just requisite, smoothed activities. Internal flow of parts was optimized, tasks were reorganized within the metrology and the planning of inspections was coordinated with the needs of the production line. We also re-examined our control policy, in particular we introduced the form measurement on CMM.
\end{abstract}

\section{Présentation du projet industriel}

Tout a commencé en 2008, année à laquelle PSA a engagé le projet du moteur 3 cylindres essence atmosphérique nommé EB dont la version turbo «PureTech»a été élue moteur de l'année dans sa catégorie par le jury du «International Engine Awards of the Year $2015 »$ le 17 juin dernier. Le site PSA de Trémery (57) a alors été désigné pour accueillir le module de production d'une capacité de 640000 moteurs par an. La particularité de ce nouvel atelier est d'avoir été entièrement conçu suivant le concept du «Lean Manufacturing» appelé en interne le «PSA Excellence System ».

Les principaux objectifs par rapport au précédent projet moteur (EP) ont alors été les suivants :

Tableau 1. Objectifs du projet EB

\begin{tabular}{|c|c|}
\hline Investissements & $-20 \%$ \\
\hline Stocks & $-30 \%$ \\
\hline Surface process & $-35 \%$ \\
\hline Lead-time & $-50 \%$ \\
\hline
\end{tabular}

La métrologie a donc été concerné par ce projet ce qui nous a amené à avoir une réflexion sur l'investissement des moyens de mesure au juste nécessaire et une activité lissée pour livrer les résultats de mesure au juste à temps.

Correspondance de l'auteur : florian.bonzon@mpsa.com

\footnotetext{
Conespondace del'areur: loian.bonzon
}

\section{La mesure au juste nécessaire}

\subsection{Mesures géométriques et d'écarts de forme}

L'axe de travail s'est concentré essentiellement sur les Machines de Mesures Tridimensionnelles (MMT) qui concentrent la grande majorité des caractéristiques contrôlées dans une métrologie. Le levier source de gain nous paraissant incontournable, fut de remplacer la mesure point à point (dit discret), employée depuis des années chez PSA, par du scanning contact. Outre la réduction des temps de contrôle, la possibilité de mesurer des écarts de forme sur MMT nous permettait de s'affranchir d'un investissement conséquent d'une machine spécifique pour un faible taux d'utilisation.

Nous avons alors entrepris une démarche de classification des MMT proposées au catalogue de notre panel fournisseurs afin de définir leur limite d'aptitude suivant notre règle d'incertitude globale tolérée fixé chez PSA à IT/8. Cette démarche s'est appuyée sur un étalon spécifique d'ondulation s'approchant $\mathrm{du}$ principe d'évaluation de l'erreur maximale permissible MPE RONt $_{\text {t }}$ (suivant la norme NF EN ISO 12181) et en utilisant la configuration palpeurs envisagée pour l'élément à mesurer le plus contraignant à savoir les fûts du cartercylindres. Elle nous a permis de choisir une machine capable de répondre à notre besoin sans pour autant retenir des machines de haute précision destinées aux laboratoires. Au final, seulement 4 MMT ont été installées et non 5 ainsi qu'un non-investissement d'une machine spécifique d'écart de forme représentant un gain d'investissement de $45 \%$ et de surface de $30 \%$ par rapport au précédent projet moteur (EP)! 


\subsection{Mesures d'états de surface}

Une étude a également été menée sur la nécessité de mesurer toutes les caractéristiques d'états de surface pour les pièces prismatiques (culasse et carter-cylindres). En effet, le fait d'avoir un atelier de fabrication avec exclusivement des machines-outils de dernière génération nous garantit un certain niveau de qualité. Nous avons donc fixé la limite à la valeur de R10 (suivant norme NF EN ISO 12085) où chaque spécification supérieure à cette valeur n'est pas mesurée pour les alésages car assurée par le process et simplement comparer à une plaquette visiotactile pour les fraisages. Au final, une réduction des caractéristiques mesurées en métrologie de $12 \%$ pour la culasse et $26 \%$ pour le carter-cylindres !

\section{Une activité lissée au juste à temps}

Un état de lieux de nos métrologies d'atelier notamment sur les modes de fonctionnement des hommes et des moyens nous a permis de constater de fortes variations de charge avec des périodes d'inactivité et des périodes de saturation suivant l'arrivée des pièces de l'atelier. Afin d'avoir une charge de travail constante, l'idée a été tout simplement de planifier les activités de la métrologie en créant un agenda hebdomadaire synchronisé avec les équipes de fabrication sur tout le temps effectif de production hebdomadaire. Des créneaux ont bien entendu été laissés volontairement libres pour prévenir de tout aléa demandant de la réactivité. Cependant, chacune des machines-outils (plus d'une centaine au total) est inscrite au programme avec une heure d'arrivée fixe assurée par les équipes de fabrication afin que ces dernières aient un délai de retour des résultats de mesure constamment court comme souhaité pour maintenir l'appareil industriel au nominal. Aussi, ce mode de fonctionnement permet de respecter le principe du FIFO respectant un flux avec un sas de mise en température des pièces en attente de mesure, les mesures tridimensionnelles puis les mesures de surface et de forme, enfin un sas de sortie pour mise à disposition des pièces mesurées.

\section{L’amélioration continue}

Toujours suivant le concept du "Lean Manufacturing », nous avons dans un premier temps cherché à standardiser la démarche sur nos derniers projets notamment la production de la version EB turbo «PureTech» sur le site de Française de Mécanique à Douvrin (62). Puis, en prévision de nos futurs projets moteurs à l'horizon 2020 , nous souhaitons continuer notre démarche sur les MMT en les implantant directement dans les lignes de fabrication afin de supprimer le transport des pièces à la métrologie pour permettre l'autonomie des équipes de fabrication dans la planification de leurs contrôles. Concernant les mesures d'états de surface, nous regardons pour descendre la valeur limite à R8 voir R6,3 et plus à R10 comme actuellement dans le but de réduire encore le nombre de caractéristiques mesurées. 